\title{
A Lie Symmetry Classification of a Nonlinear Fin Equation in Cylindrical Coordinates
}

\author{
Saeed M. Ali, Ashfaque H. Bokhari, and F. D. Zaman \\ Department of Mathematics and Statistics, King Fahd University of Petroleum \& Minerals, Dhahran 31261, Saudi Arabia \\ Correspondence should be addressed to Ashfaque H. Bokhari; abokhari@kfupm.edu.sa
}

Received 7 April 2014; Accepted 20 May 2014; Published 26 June 2014

Academic Editor: Rehana Naz

Copyright (C) 2014 Saeed M. Ali et al. This is an open access article distributed under the Creative Commons Attribution License, which permits unrestricted use, distribution, and reproduction in any medium, provided the original work is properly cited.

\begin{abstract}
The nonlinear fin equation in cylindrical coordinates is considered. Assuming a radial variable heat transfer coefficient and temperature dependent thermal conductivity, a complete classification of these two functions is obtained via Lie symmetry analysis. Using these Lie symmetries, we carry out reduction of the fin equation and whenever possible exact solutions are obtained.
\end{abstract}

\section{Introduction}

A heat exchange in industrial applications such as compressors, air conditioners, and air craft engines is achieved through the surfaces called fins. Fins which are of different shapes are described by a variety of mathematical models [1]. Moitsheki [2] has recently discussed the problem of temperature profiles and heat transfer per fin length by considering 2-dimensional Laplace equation given in the following form:

$$
\frac{\partial^{2} \theta}{\partial x^{2}}+\frac{\partial^{2} \theta}{\partial y^{2}}=0
$$

In this problem, the author uses the method of separation of variables and the Newton Raphson method for computing the temperature profile and heat transfer. More recently, Pakdemirli and Sahin $[3,4]$ have studied the problem

$$
\frac{\partial}{\partial x}\left(k(\theta) \frac{\partial \theta}{\partial x}\right)-N^{2} f(x) \theta=\theta_{t}
$$

where $k(\theta)$ is conductivity, $N$ is the fin constant, and $f(x)$ is heat transfer coefficient using the Lie symmetries of the above governing partial differential equation (2). This method was introduced by Lie [5] to find exact solutions of a number of linear and nonlinear PDEs arising in engineering, mathematical, and biological sciences. A good account of the Lie approach and its applications to the differential equations can be found in [6-9]. Bokhari et al. [10] have recently studied the above nonlinear fin equation (2) using Lie symmetry approach and give some new interesting exact solutions. Moitsheki et al. [11] obtained some exact solutions of (2) by considering a power law form of the thermal conductivity. Moitsheki [2] has also considered a radial onedimensional steady state heat transfer problem by assuming the fin equation in the following form:

$$
\frac{A_{p}}{r} \frac{d}{d r}\left(r f(r) k(u) \frac{d u}{d r}\right)=\phi(u)\left(u-u_{a}\right), \quad r_{b}<r<r_{a},
$$

where $k$ and $h$ are the nonuniform thermal conductivity and heat transfer coefficients, respectively, depending on the temperature. Some exact solutions are obtained for thermal diffusion fin with a rectangular profile and a hyperbolic profile. Continuing their investigation, Moitsheki [12] has also studied a steady heat transfer problem of a longitudinal fin with triangular and parabolic shapes by considering the following problem:

$$
A_{p} \frac{d}{d x}\left(F(x) k(u) \frac{d u}{d x}\right)=\phi(u)\left(u-u_{a}\right), \quad 0<x<L,
$$

where $A_{p}$ represents the profile area, $F(x)$ represents fin profile, and $k$ and $h$, respectively, represent nonuniform thermal conductivity and heat transfer coefficient depending on the temperature. 
Vaneeva et al. [13] have performed a Lie group classification of the nonlinear $(1+1)$ dimensional fin equation and presented exact solutions considering the steady state heat transfer problem for a rectangular fin. Moitsheki and Rowjee [14] have discussed the $(1+1)$ dimensional problem

$$
\frac{\partial}{\partial y}\left(k(u) \frac{\partial u}{\partial y}\right)+\frac{\partial}{\partial x}\left(k(u) \frac{\partial u}{\partial x}\right)=s(u)
$$

in which $u$ is the dimensionless temperature, $s$ is the internal heat generation function, and $k$ is the thermal conductivity. Employing the Lie symmetry analysis to classify the internal heat generating function, they obtained some reductions of the fin equation as well as exact solutions. Some authors have also considered the two-dimensional problem with $s=0$ in (5) by assuming constant thermal conductivity $[15,16]$. In the same series of studies Moitsheki and Harley [17] have considered a two-dimensional pin fin equation with length $L$ and radius $R$, having the form

$$
\frac{1}{R} \frac{\partial}{\partial R}\left(R k(u) \frac{\partial u}{\partial R}\right)+\frac{\partial}{\partial x}\left(k(u) \frac{\partial u}{\partial x}\right)=s(u) .
$$

Using Lie symmetry approach, they give certain solutions of this equation for different cases of $s(u)$. Extending this work in this area, we present a complete classification of the nonlinear $(2+1)$ fin equation by considering cylindrical fins with a temperature dependent thermal conductivity and variable heat transfer coefficient. The fin equation, in this case, can be written as

$$
\frac{1}{x} \frac{\partial}{\partial x}\left(x k(u) u_{x}\right)+\frac{1}{x} \frac{\partial}{\partial y}\left(\frac{1}{x} k(u) u_{y}\right)-N^{2} f(x) u=u_{t},
$$

which can be rewritten in the form

$$
\begin{gathered}
x^{2} k(u) u_{x x}+x^{2} k(u)_{u} u_{x}^{2}+x k(u) u_{x}+k(u)_{u} u_{y}^{2} \\
+k(u) u_{y y}-x^{2} N^{2} f(x) u-x^{2} u_{t}=0,
\end{gathered}
$$

where $x$ and $y$, respectively, represent radial and angular coordinates. Also $u, k, f$, and $N$ in (8), respectively, represent the dimensionless temperature, the thermal conductivity, the heat transfer, and the fin parameter. Using Lie symmetry analysis, we present a complete classification of $k(u)$ and $f(x)$ and obtain exact solutions in cases of interest. The results presented in this paper are more general than previously studied cases in the literature [2-4, 10-12, 14-17]. The plan of this paper is as follows. In the next section, we perform a complete symmetry analysis of the fin equation. In Section 3 , we present classification of $k$ and $f$ according to Lie point symmetries. In Section 4, we present exact solutions whenever possible and in other cases reduce the fin equation to ODE. A brief summary of this work is given in the last section.

\section{Symmetry Analysis of the Fin Equation}

In order to classify solutions of the Fin equation, we use the well-known Lie symmetry method [8]. This method is based upon finding Lie point symmetries of the PDEs that leave them invariant. In order to derive symmetry generators of the Fin equation, we consider one parameter Lie point transformation that leaves it invariant. The transformation [16]

$$
\tilde{x}^{i}=x^{i}+\varepsilon \xi^{i}(x, y, t, u)+O\left(\varepsilon^{2}\right), \quad i=1, \ldots, 4,
$$

where $\xi^{i}=\partial \tilde{x}^{i} /\left.\partial \varepsilon\right|_{\varepsilon=0}$ defines the symmetry generator associated with (9) given by

$$
X=\xi \frac{\partial}{\partial x}+\eta \frac{\partial}{\partial y}+\tau \frac{\partial}{\partial t}+\phi \frac{\partial}{\partial u}
$$

The prolonged symmetry generator associated with (8) has the following form:

$$
\begin{aligned}
\mathbf{X}^{(2)}= & X+\sum_{I=0}^{2} \phi^{J} \frac{\partial}{\partial u_{J}} \\
& +\sum_{I, J=0}^{2} \phi^{I J} \frac{\partial}{\partial u_{I J}}, \quad I, J=0, \ldots, 2,
\end{aligned}
$$

where 0 represents $t$ and 1 and 2, respectively, represent $x$ and $y$, and the coefficients, $\phi^{J}$ and $\phi^{J K}$, of the derivatives with respect to dependent variables in (11) are evaluated using the expressions

$$
\begin{gathered}
\phi^{J}=D_{i}\left(\phi-\xi^{j} u_{j i}\right)+\xi^{j} u_{j, i}, \\
\phi^{J K}=D_{i} D_{j}\left(\phi-\xi^{j} u_{j i}\right)+\xi^{k} u_{k, i j} .
\end{gathered}
$$

At this stage we use the Lie symmetry criterion by requiring that $(8)$ is invariant under the prolonged symmetry generator given in (11) modulu Equation (8) itself. Mathematically, this requirement is given by

$$
\begin{aligned}
& \mathbf{X}^{(2)}\left[x^{2} k(u) u_{x x}+x^{2} k(u)_{u} u_{x}^{2}+x k(u) u_{x}\right. \\
& \left.\quad+k(u)_{u} u_{y}^{2}+k(u) u_{y y}-x^{2} N^{2} f(x) u-x^{2} u_{t}\right]\left.\right|_{(8)}=0 .
\end{aligned}
$$

Using (12) and comparing terms involving derivatives of the dependent function $u$, we obtain the following determining equations:

$$
\begin{gathered}
\xi_{u}=0=\eta_{u}=\tau_{u}=\phi_{u u}=\tau_{y}=\tau_{x}, \\
x^{2} \eta_{t}-k(u) x \eta_{x}-k(u) x^{2} \eta_{x x}-k(u) \eta_{y y}+2 k(u) \phi_{u y}=0, \\
-k(u) \xi+x \phi(k(u))_{u}-k(u) x \xi_{x}+k(u) x \tau_{t} \\
+x^{2} \xi_{t}+2 k(u) x^{2} \phi_{x u}-k(u) x^{2} \xi_{x x}-k(u) \xi_{y y}=0,
\end{gathered}
$$




$$
\begin{gathered}
f(x) N^{2} x^{2} \phi+N^{2} u x^{2} \xi(f(x))_{x}+x^{2} \phi_{t} \\
-f(x) N^{2} u x^{2} \phi_{u}-k(u) x \phi_{x}+f(x) N^{2} u x^{2} \tau_{t} \\
-k(u) x^{2} \phi_{x x}-k(u) \phi_{y y}=0 \\
x^{2} \eta_{x}+\xi_{y}=0 \\
\phi k(u)_{u}-2 k(u) \xi_{x}+k(u) \tau_{t}=0 \\
-2 k(u) \xi+x \phi k(u)_{u}-2 x k(u) \eta_{y}+k(u) x \tau_{t}=0
\end{gathered}
$$

To determine the unknown functions, $\eta, \tau$, and $\phi$, we solve the above coupled system of differential equations by first considering (19). Differentiating this equation twice with respect to $u$ leads to the following expression:

$$
\phi_{u u}=\left(\frac{k}{k_{u}}\right)_{u u}\left(2 \xi_{x}-\tau_{t}\right) .
$$

Using (14) into (21), it reduces to

$$
\left(\frac{k}{k_{u}}\right)_{u u}\left(2 \xi_{x}-\tau_{t}\right)=0 .
$$

We proceed from above equation to obtain complete classification of both $k$ and $f$ as shown in the next section.

\section{Classification}

In order to find a complete classification of solutions of (8), we note that the following three cases arise from (22):

$$
\begin{aligned}
& \text { (1) }\left(k / k_{u}\right)_{u u}=0, \\
& \text { (2) } 2 \xi_{x}-\tau_{t}=0, \\
& \text { (3) } 2 \xi_{x}-\tau_{t}=0=\left(k / k_{u}\right)_{u u} \text {. }
\end{aligned}
$$

For obtaining a complete classification, we consider all the three cases one by one. Since procedure of classification in all the three cases is similar, we give detailed procedure in the first case and only give results in the remaining cases. To begin the classification, we proceed as follows.

Case 1. Solving equation $\left(k / k_{u}\right)_{u u}=0$, for $k(u)$ instantly yields

$$
k(u)=\gamma(\alpha u+\beta)^{1 / \alpha}
$$

where $\gamma, \alpha$, and $\beta$ are integration constants. Using (23) into (19) immediately gives

$$
\phi=(\alpha u+\beta)\left(2 \xi_{x}-\tau_{t}\right) .
$$

Using (23) and (24) in (20), we obtain a differential relation in $\xi$ and $\eta$ given by

$$
\eta_{y}=\xi_{x}-\frac{1}{x} \xi
$$

Differentiating (24) twice and (25) once with respect to $y$ and using (18) in the resulting expressions give

$$
\begin{gathered}
\phi_{u y}=-2 \alpha x^{2} \eta_{x x}-4 \alpha x \eta_{x} \\
\eta_{y y}=-x^{2} \eta_{x x}-x \eta_{x} .
\end{gathered}
$$

At this stage we use (27) and (26) into (20), to get

$$
x^{2} \eta_{t}-4 \alpha k x^{2} \eta_{x x}-8 \alpha k x \eta_{x}=0 .
$$

Differentiating (28) with respect to " $u$," while keeping (23) in mind, we obtain

$$
-4 \alpha \gamma(\alpha u+\beta)\left(\frac{1}{\alpha}-1\right) x\left[x \eta_{x x}+2 \eta_{x}\right]=0 .
$$

Keeping in mind that $\alpha, \beta$, and $\gamma$ in the above equation are nonzero, we conclude that the above equation is satisfied only when

$$
x \eta_{x x}+2 \eta_{x}=0
$$

(the case $\alpha=1$ is not to be considered as it becomes a special case of (1.3) that is dealt with later). Note that (30) is a separable DE and can be easily solved to find $\eta$ given by

$$
\eta(x, y, t)=-c(y, t) x^{-1}+d(y, t) .
$$

To require consistency of $\eta$ found above, we use (31) into (28). This suggests that (28) is satisfied when the following differential constraint is met:

$$
-c_{t}(y, t)+x d_{t}(y, t)=0
$$

The above equation implies that $d_{t}(y, t)=\gamma(y)$ and $c(y, t)=$ $\lambda(y)$. Using these results into (32) gives

$$
\eta(x, y, t)=-\lambda(y) x^{-1}+\gamma(y) .
$$

To determine $\gamma$ we use (33) into (27). This leads to the following second-order differential constraint:

$$
x \gamma_{y y}(y)-\lambda_{y y}(y)-\lambda(y)=0
$$

To solve the above equation, we differentiate it with respect to $x$ to get

$$
\gamma(y)=c_{1} y+c_{2}
$$

We then put (35) into (34), to get a second-order linear differential equation,

$$
\lambda_{y y}(y)+\lambda(y)=0 .
$$

The above equation is solved to get

$$
\lambda(y)=c_{3} \cos y+c_{4} \sin y .
$$

At this stage we use (35) and (37) into (33), to infer that

$$
\eta(x, y)=\frac{-1}{x}\left[c_{3} \cos y+c_{4} \sin y\right]+c_{1} y+c_{2} \text {. }
$$


Having determined $\eta$ completely, we now find $\xi$. For this purpose, we use (38) there. This yields an expression for $\xi$ as given below:

$$
\xi(x, y, t)=-c_{3} \sin y+c_{4} \cos y+\gamma_{1}(x, t) .
$$

To determine $\gamma_{1}$, we use (39) and (38) into (25) to obtain

$$
\gamma_{1}(x, t)=c_{1} x \ln x+x \lambda_{1}(t) .
$$

Using above value of $\gamma_{1}$ into (39) and (24), respectively, becomes

$$
\begin{gathered}
\xi(x, y, t)=-c_{3} \sin y+c_{4} \cos y+c_{1} x \ln x+x \lambda_{1}(t), \\
\phi=(\alpha u+\beta)\left(2 c_{1}+2 c_{1} \ln x+2 \lambda_{1}(t)-\tau_{t}\right) .
\end{gathered}
$$

We now use all the above results into (4), which suggests that it is satisfied if the following condition is met:

$$
x^{3} \lambda_{1}(t)+4 \gamma \alpha c_{1}(\alpha u+\beta)^{1 / \alpha}=0 .
$$

From the above equation, we immediately infer that $\lambda_{1}(t)=$ $c_{5}$ and $c_{1}=0$. Therefore, (38), (41), and (42) take the form

$$
\begin{gathered}
\xi(x, y, t)=-c_{3} \sin y+c_{4} \cos y+c_{5} x \\
\eta(x, y)=\frac{-1}{x}\left[c_{3} \cos y+c_{4} \sin y\right]+c_{2} \\
\phi=(\alpha u+\beta)\left(2 c_{5}-\tau_{t}\right) .
\end{gathered}
$$

To determine consistency, we now use the last of the determining equations, that is, (17). This requirement gives

$$
\begin{aligned}
& \beta f(x) N^{2}\left(2 c_{5}-\tau_{t}\right)+N^{2} u f_{x}\left(-c_{3} \sin y+c_{4} \cos y+c_{5} x\right) \\
& -(\alpha u+\beta) \tau_{t t}+f N^{2} u \tau_{t}=0 .
\end{aligned}
$$

In order for (45) to be satisfied, we proceed as follows: comparing coefficients of $f(x)$, this equation gives

$$
-\beta f(x) N^{2} \tau_{t t}-(\alpha u+\beta) \tau_{t t t}+f(x) N^{2} u \tau_{t t}=0 .
$$

Differentiation of the above equation with respect to $u$ gives

$$
-\alpha \tau_{t t t}+f(x) N^{2} \tau_{t t}=0 .
$$

The above equation is a separable DE in $x$ and $t$ and can be written as

$$
\frac{\tau_{t t t}}{\tau_{t t}}=\frac{f(x) N^{2}}{\alpha} ;
$$

solving (48) implies that $f(x)=c$ ( $c$ a constant) whereas the $\tau$ becomes

$$
\tau(t)=\frac{c_{6} \alpha^{2}}{c^{2} N^{4}} \exp \left(\frac{c N^{2}}{\alpha} t\right)+c_{7} t+c_{8} .
$$

To require consistency, we use (49) with $f(x)=c \neq 0$ in (46), to obtain

$$
\beta c_{6}(\alpha+1)=0
$$

From (50) four cases arise, namely,

$$
\begin{aligned}
& \text { (1.1) } \alpha=-1, \beta \neq 0, c_{6} \neq 0, \\
& \text { (1.2) } \alpha \neq-1, \beta=0, c_{6} \neq 0, \\
& \text { (1.3) } \alpha \neq-1, \beta \neq 0, c_{6}=0, \\
& \text { (1.4) } \alpha=-1, \beta=0, c_{6}=0 .
\end{aligned}
$$

We first consider Case 1.1

Case $1.1(k(u)=\gamma /(\beta-u)$ and $f(x)=c)$. Using these conditions arising in this case into ((49), (45), and (44)) the infinitesimal symmetry generators $\xi, \eta, \tau, \phi$, and $k$ are determined as

$$
\begin{aligned}
\xi & =-c_{3} \sin y+c_{4} \cos y, \\
\eta & =-c_{3} \frac{\cos y}{x}-c_{4} \frac{\sin y}{x}+c_{2}, \\
\tau & =\frac{c_{6}}{c^{2} N^{4}} \exp \left(-c N^{2} t\right)+c_{8}, \\
\phi & =\frac{c_{6}}{c N^{2}}(-u+\beta) \exp \left(-c N^{2} t\right) .
\end{aligned}
$$

The five symmetry generators associated with above infinitesimals are given by

$$
\begin{aligned}
& X_{1}=-\sin y \frac{\partial}{\partial x}-\frac{\cos y}{x} \frac{\partial}{\partial y}, \\
& X_{2}=\cos y \frac{\partial}{\partial x}-\frac{\sin y}{x} \frac{\partial}{\partial y}, \\
& X_{3}=\frac{1}{c^{2} N^{4}} \exp \left(-c N^{2} t\right) \frac{\partial}{\partial t}+\frac{\beta-u}{c N^{2}} \exp \left(-c N^{2} t\right) \frac{\partial}{\partial u}, \\
& X_{4}=\frac{\partial}{\partial t}, \\
& X_{5}=\frac{\partial}{\partial y} .
\end{aligned}
$$

The commutation relations for each of the above symmetry generators are listed in Table 1.

Case $1.2\left(k(u)=\gamma(\alpha u)^{1 / \alpha}\right.$ and $\left.f(x)=c\right)$. Using the values of $k$ and $f$ of this case into (49), (45), and (44), the expressions for $\xi, \eta, \tau$, and $\phi$ take the forms

$$
\begin{aligned}
& \xi=-c_{3} \sin y+c_{4} \cos y+c_{5} x, \\
& \eta=-c_{3} \frac{\cos y}{x}-c_{4} \frac{\sin y}{x}+c_{2}, \\
& \tau=\frac{c_{6} \alpha^{2}}{c^{2} N^{4}} \exp \left(-c N^{2} t\right)+c_{8}, \\
& \phi=\alpha u\left(2 c_{5}-\frac{c_{6} \alpha}{c N^{2}} \exp \left(-c N^{2} t\right)\right) .
\end{aligned}
$$


TABLE 1: Case 1.1: commutation relations satisfied by symmetry generators.

\begin{tabular}{lccccc}
\hline$\left[X_{i}, X_{j}\right]$ & $X_{1}$ & $X_{2}$ & $X_{3}$ & $X_{4}$ & $X_{5}$ \\
\hline$X_{1}$ & 0 & 0 & 0 & 0 & $X_{2}$ \\
$X_{2}$ & 0 & 0 & 0 & 0 & $X_{1}$ \\
$X_{3}$ & 0 & 0 & 0 & $c N^{2} X_{3}$ & 0 \\
$X_{4}$ & 0 & 0 & $-c N^{2} X_{3}$ & 0 & 0 \\
$X_{5}$ & $-X_{2}$ & $-X_{1}$ & 0 & 0 & 0 \\
\hline
\end{tabular}

TABle 2: Commutation relations in Case 1.2.

\begin{tabular}{lcccccc}
\hline$\left[X_{i}, X_{j}\right]$ & $X_{1}$ & $X_{2}$ & $X_{3}$ & $X_{4}$ & $X_{5}$ & $X_{6}$ \\
\hline$X_{1}$ & 0 & 0 & $X_{1}$ & 0 & 0 & $X_{2}$ \\
$X_{2}$ & 0 & 0 & $X_{2}$ & 0 & 0 & $-X_{1}$ \\
$X_{3}$ & $-X_{1}$ & $-X_{2}$ & 0 & 0 & 0 & 0 \\
$X_{4}$ & 0 & 0 & 0 & 0 & $\frac{c N^{2}}{\alpha} X_{4}$ & 0 \\
$X_{5}$ & 0 & 0 & 0 & $\frac{-c N^{2}}{\alpha} X_{4}$ & 0 & 0 \\
$X_{6}$ & $-X_{2}$ & $X_{1}$ & 0 & 0 & 0 & 0 \\
\hline
\end{tabular}

Accordingly, the six symmetry generators associated with above infinitesimals are given by

$$
\begin{aligned}
& X_{1}=-\sin y \frac{\partial}{\partial x}-\frac{\cos y}{x} \frac{\partial}{\partial y}, \\
& X_{2}=\cos y \frac{\partial}{\partial x}-\frac{\sin y}{x} \frac{\partial}{\partial y}, \\
& X_{3}=x \frac{\partial}{\partial x}+2 \alpha u \frac{\partial}{\partial u}, \\
& X_{4}=\frac{\alpha^{2}}{c^{2} N^{4}} \exp \left(\frac{c N^{2}}{\alpha} t\right) \frac{\partial}{\partial t}-\frac{\alpha^{2} u}{c N^{2}} \exp \left(\frac{c N^{2}}{\alpha} t\right) \frac{\partial}{\partial u}, \\
& X_{5}=\frac{\partial}{\partial t}, \\
& X_{6}=\frac{\partial}{\partial y} .
\end{aligned}
$$

The commutation relations for these generators are given in Table 2.

Case $1.3\left(k(u)=\gamma(\alpha u+\beta)^{1 / \alpha}\right.$ and $\left.f(x)=c\right)$. Using the conditions with the values of $k$ and $f$ of this case into (49), (45), and (44), the expressions for $\xi, \eta, \tau$, and $\phi$ take the forms

$$
\begin{gathered}
\xi=-c_{3} \sin y+c_{4} \cos y, \quad \eta=-c_{3} \frac{\cos y}{x}-c_{4} \frac{\sin y}{x}+c_{2}, \\
\tau=c_{8}, \quad \phi=0
\end{gathered}
$$

TABLE 3: Commutation relations in Case 1.3.

\begin{tabular}{lcccc}
\hline$\left[X_{i}, X_{j}\right]$ & $X_{1}$ & $X_{2}$ & $X_{3}$ & $X_{4}$ \\
\hline$X_{1}$ & 0 & 0 & 0 & $X_{2}$ \\
$X_{2}$ & 0 & 0 & 0 & $-X_{1}$ \\
$X_{3}$ & 0 & 0 & 0 & 0 \\
$X_{4}$ & $-X_{2}$ & $X_{1}$ & 0 & 0 \\
\hline
\end{tabular}

and the corresponding generators are

$$
\begin{aligned}
& X_{1}=-\sin y \frac{\partial}{\partial x}-\frac{\cos y}{x} \frac{\partial}{\partial y} \\
& X_{2}=\cos y \frac{\partial}{\partial x}-\frac{\sin y}{x} \frac{\partial}{\partial y} \\
& X_{3}=\frac{\partial}{\partial t} \\
& X_{4}=\frac{\partial}{\partial y}
\end{aligned}
$$

As before the commutation relations form a closed algebra and are represented in Table 3.

Case $1.4(k(u)=-\gamma / u$ and $f(x)=c)$. Similarly, using the conditions with the values of $k$ and $f$ of this case into (49), (45), and (44), the expressions for $\xi, \eta, \tau$, and $\phi$ take the forms

$$
\begin{gathered}
\xi=-c_{3} \sin y+c_{4} \cos y+c_{5} x, \\
\eta=-c_{3} \frac{\cos y}{x}-c_{4} \frac{\sin y}{x}+c_{2}, \\
\tau=c_{8}, \quad \phi=-2 c_{5} u .
\end{gathered}
$$

With the above infinitesimals there are five generators associated:

$$
\begin{aligned}
& X_{1}=-\sin y \frac{\partial}{\partial x}-\frac{\cos y}{x} \frac{\partial}{\partial y} \\
& X_{2}=\cos y \frac{\partial}{\partial x}-\frac{\sin y}{x} \frac{\partial}{\partial y} \\
& X_{3}=x \frac{\partial}{\partial x}-2 u \frac{\partial}{\partial u} \\
& X_{4}=\frac{\partial}{\partial t} \\
& X_{5}=\frac{\partial}{\partial y}
\end{aligned}
$$

The commutation relation satisfied by the above five generators is given in Table 4 . 
TABLE 4: Commutation relations in Case 1.4.

\begin{tabular}{lccccc}
\hline$\left[X_{i}, X_{j}\right]$ & $X_{1}$ & $X_{2}$ & $X_{3}$ & $X_{4}$ & $X_{5}$ \\
\hline$X_{1}$ & 0 & 0 & $X_{1}$ & 0 & $X_{2}$ \\
$X_{2}$ & 0 & 0 & $X_{2}$ & 0 & $-X_{1}$ \\
$X_{3}$ & $-X_{1}$ & $-X_{2}$ & 0 & 0 & 0 \\
$X_{4}$ & 0 & 0 & 0 & 0 & 0 \\
$X_{5}$ & $-X_{2}$ & $X_{1}$ & 0 & 0 & 0 \\
\hline
\end{tabular}

Case $2\left(2 \xi_{x}-\tau_{t}=0\right)$. In this case the system of determining equations given by (14)-(20) becomes

$$
\begin{gathered}
\xi_{u}=0=\eta_{u}=\tau_{u}=\phi=\tau_{y}=\tau_{x}, \\
x^{2} \eta_{t}-k(u) x \eta_{x}-k(u) x^{2} \eta_{x x}-k(u) \eta_{y y}=0, \\
-k(u) \xi+k(u) x \xi_{x}+x^{2} \xi_{t}-k(u) x^{2} \xi_{x x}-k(u) \xi_{y y}=0, \\
N^{2} u x^{2} \xi(f(x))_{x}+2 f(x) N^{2} u x^{2} \xi_{x}=0, \\
x^{2} \eta_{x}+\xi_{y}=0, \\
-2 k(u) \xi-2 x k(u) \eta_{y}+2 k(u) x \xi_{x}=0 .
\end{gathered}
$$

Following the procedure adopted in Case 1, we easily find that

$$
\xi=c_{1} x, \quad \eta=c_{3}, \quad \tau=2 c_{1}+c_{2}, \quad \phi=0 .
$$

The above infinitesimals satisfy all the equations in the system (59) except (iv). Using (60) in (59)-(iv), we obtain,

$$
c_{1} N^{2} x^{3} u f_{x}+2 c_{1} N^{2} x^{2} u f=0 .
$$

From (61) two cases arise:

$$
\begin{aligned}
& \text { (2.1) } c_{1}=0 \\
& \text { (2.2) } c_{1} \neq 0 .
\end{aligned}
$$

Case 2.1. In this case $k(u)$ and $f(x)$ in system (59) are arbitrary functions and the general expressions of $\xi, \eta, \tau$, and $\phi$ take the forms

$$
\xi=0, \quad \eta=c_{4}, \quad \tau=c_{5}, \quad \phi=0 .
$$

The two commuting symmetry generators in this case are

$$
X_{1}=\frac{\partial}{\partial y}, \quad X_{2}=\frac{\partial}{\partial t} .
$$

Case 2.2. Here $k(u)$ is an arbitrary function and $f(x)=c / x^{2}$. The general expressions of $\xi, \eta, \tau$, and $\phi$ are

$$
\tau=2 c_{1} t+c_{2}, \quad \xi=c_{1} x, \quad \eta=c_{3}, \quad \phi=0 .
$$

TABLE 5: Commutation relations in Case 2.2.

\begin{tabular}{lccc}
\hline$\left[X_{i}, X_{j}\right]$ & $X_{1}$ & $X_{2}$ & $X_{3}$ \\
\hline$X_{1}$ & 0 & $-2 X_{2}$ & 0 \\
$X_{2}$ & $2 X_{2}$ & 0 & 0 \\
$X_{3}$ & 0 & 0 & 0 \\
\hline
\end{tabular}

The three symmetry generators associated with (64) are

$$
X_{1}=x \frac{\partial}{\partial x}+2 t \frac{\partial}{\partial t}, \quad X_{2}=\frac{\partial}{\partial t}, \quad X_{3}=\frac{\partial}{\partial y}
$$

The commutation relation satisfied by three generators is presented in Table 5.

Case $3\left(2 \xi_{x}-\tau_{t}=0=\left(k / k_{u}\right)_{u u}\right)$. This case gives

$$
k(u)=\gamma(\alpha u+\beta)^{1 / \alpha}, \quad \phi=0, \quad \tau_{t}=2 \xi_{x} .
$$

Consequently, the system of determining equations given by (14)-(20) becomes

$$
\begin{gathered}
\xi_{u}=0=\eta_{u}=\tau_{u}=\phi=\tau_{y}=\tau_{x}, \\
x^{2} \eta_{t}-\gamma(\alpha u+\beta)^{1 / \alpha} x \eta_{x}-\gamma(\alpha u+\beta)^{1 / \alpha} x^{2} \eta_{x x} \\
-\gamma(\alpha u+\beta)^{1 / \alpha} \eta_{y y}=0,
\end{gathered}
$$$$
-\gamma(\alpha u+\beta)^{1 / \alpha} \xi+\gamma(\alpha u+\beta)^{1 / \alpha} x \xi_{x}+x^{2} \xi_{t}
$$$$
-\gamma(\alpha u+\beta)^{1 / \alpha} x^{2} \xi_{x x}-\gamma(\alpha u+\beta)^{1 / \alpha} \xi_{y y}=0,
$$$$
N^{2} u x^{2} \xi(f(x))_{x}+2 f(x) N^{2} u x^{2} \xi_{x}=0,
$$$$
x^{2} \eta_{x}+\xi_{y}=0
$$

$$
\begin{aligned}
& -2 \gamma(\alpha u+\beta)^{1 / \alpha} \xi-2 x \gamma(\alpha u+\beta)^{1 / \alpha} \eta_{y} \\
& +2 \gamma(\alpha u+\beta)^{1 / \alpha} x \xi_{x}=0 .
\end{aligned}
$$

Following the procedure adopted in earlier cases for the system (67), we obtain same symmetry generators in Cases 2 and 3. The difference though is that in Case $2 k(u)$ is arbitrary while in Case $3 k(u)=\gamma(\alpha u+\beta)^{1 / \alpha}$.

\section{Reduction under Two-Dimensional Subalgebra and Exact Invariant Solutions}

Case 1. In this section, we present solutions of (8) via reductions. These reductions are obtained by the similarity variables obtained through symmetry generators. To perform reductions of (8), we first consider two symmetry generators from Table 1, $\mathbf{X}_{1}$ and $\mathbf{X}_{2}$, that span an abelian subalgebra. 
To start reduction, we first consider $\mathbf{X}_{1}$. The characteristic equation corresponding to this generator is

$$
\frac{d x}{-\sin y}=\frac{-x d y}{\cos y}=\frac{d t}{0}=\frac{d u}{0} .
$$

Solving the above equation, it is straight forward [6] to find that it yields the similarity variables $r=x \cos y$ and $s=t$ with $w(r, s)=u$. Replacing $u$ in (8) in terms of new variables becomes

$$
\frac{\gamma}{\beta-w} w_{r r}+\frac{\gamma}{(\beta-w)^{2}} w_{r}^{2}-N^{2} c w-w_{s}=0 .
$$

To proceed further, we first transform $\mathbf{X}_{2}$ in terms of new variables $r, s$, and $w$. Thus, $\widehat{\mathbf{X}}_{2}=\partial / \partial r$. The similarities corresponding to this generator are $z=s$ and $v(z)=w$. This reduces (69) to a first-order differential equation given by

$$
v_{z}+N^{2} c v=0
$$

Solving this equation, we immediately find that $v(z)=$ $\exp \left(-N^{2} c z\right)$, which in original coordinates becomes

$$
u(x, y, t)=\exp \left(-N^{2} c t\right) \text {. }
$$

Case 2. Here, we first consider the generators $\mathbf{X}_{1}$ and $\mathbf{X}_{3}$ given in Table 3 , satisfying $\left[\mathbf{X}_{1}, \mathbf{X}_{3}\right]=0$. Following procedure followed in the previous case, the generator $\mathbf{X}_{1}$ reduces (8) to (69). In the light of $\mathbf{X}_{1}$, the $\mathbf{X}_{3}$ transforms to $\widehat{\mathbf{X}}_{3}=$ $\left(1 / c^{2} N^{4}\right) \exp \left(-c N^{2} s\right)(\partial / \partial s)+\left(\beta-w / c N^{2}\right) \exp \left(-c N^{2} s\right)(\partial / \partial w)$, which gives $z=r$ with $w=\beta-\exp \left(-c N^{2} s\right) v(z)$. In the light of these similarity variables, (69) reduces to the following ODE:

$$
v_{z z}-\frac{1}{v} v_{z}^{2}+\frac{N^{2} c \beta}{\gamma} v=0 .
$$

Choosing $\gamma=N^{2} c \beta$, the above solution takes the form

$$
v(z)=c_{2} \exp \left(c_{1} z-\frac{1}{2} z^{2}\right) .
$$

Writing above in original coordinates, it becomes

$$
u(x, y, t)=\beta-c_{2} \exp \left(-N^{2} c t\right) \exp \left(c_{1} x \cos y-\frac{1}{2} x^{2} \cos ^{2} y\right)
$$

The graphical profile of the above solution is given in Figure 1.

For constant $t$ the same solution is plotted and the solution depicts a saddle point behavior as shown in Figure 2.

Case 3. In this case, we consider the two generators $\mathbf{X}_{3}$ and $\mathbf{X}_{6}$ that satisfy $\left[\mathbf{X}_{3}, \mathbf{X}_{6}\right]=0$ as shown in Table 2 . Since the two generators commute, we can start reduction by either $\mathbf{X}_{3}$ or $\mathbf{X}_{6}$. First considering $\mathbf{X}_{3}$, the characteristic equation becomes

$$
\frac{d x}{x}=\frac{d y}{0}=\frac{d t}{0}=\frac{d u}{2 \alpha u} .
$$

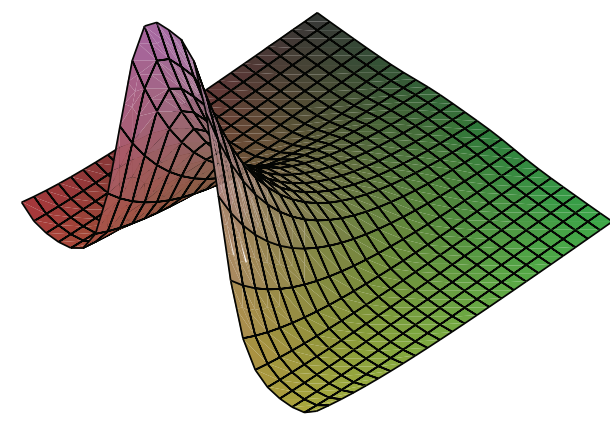

FIGURE 1

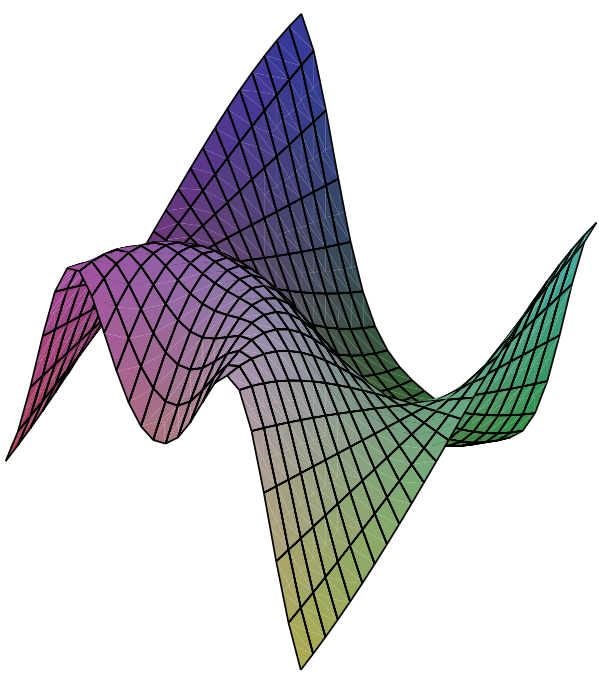

Figure 2

The similarity variables corresponding to above equation become $r=y, s=t$, and $u=x^{2 \alpha} w$. These variables reduce (8) to a PDE of the form

$$
\begin{aligned}
& 4 \gamma \alpha^{(1 / \alpha)+1}(\alpha+1) w^{(1 / \alpha)+1}+\gamma \alpha^{(1 / \alpha)-1} w^{(1 / \alpha)-1} w_{r}^{2} \\
& +\gamma \alpha^{1 / \alpha} w^{1 / \alpha} w_{r r}-N^{2} c w-w_{s}=0 .
\end{aligned}
$$

Using similarity variables transformation obtained from $\mathbf{X}_{3}$, $\mathbf{X}_{6}$ transforms to $\widehat{\mathbf{X}}_{6}=\partial / \partial r$. This leads to the new coordinates $s=z$ and $v(z)=w$. In the light of these similarities, (76) transforms to

$$
4 \gamma \alpha^{(1 / \alpha)+1}(\alpha+1) v^{(1 / \alpha)+1}-N^{2} c v-v_{z}=0 .
$$

Choosing $N^{2} c=1, \gamma=1$, and $\alpha=-1$, the above equation takes the form

$$
v_{z}+2 v=0
$$

giving exact solution $u(x, y, t)=\exp 2 t$. 
Case 4. Here, we consider the two generators $\mathbf{X}_{3}, \mathbf{X}_{4}$ given in Table 4 that satisfy $\left[\mathbf{X}_{3}, \mathbf{X}_{4}\right]=0$. First considering $X_{3}$ and its characteristic equation

$$
\frac{d x}{x}=\frac{d y}{0}=\frac{d t}{0}=\frac{d u}{-2 u} .
$$

This gives similarity variables $r=y, s=t$, and $u=x^{-2} w$. These variables reduce (8) to a PDE given below:

$$
\gamma w^{-2} w_{r}^{2}-\gamma w^{-1} w_{r r}-N^{2} c w-w_{s}=0
$$

To reduce the above equation further, we use $X_{3}$, to transform $X_{4}$ to $\widehat{X}_{4}=\partial / \partial s$. This leads to the similarity variables $r=z$ and $v(z)=w$. Using these similarities, (80) becomes an ODE,

$$
v_{z z}-v^{-1} v_{z}^{2}+\frac{N^{2} c}{\gamma} v^{2}=0
$$

Choosing $\gamma=N^{2} c$, (81) can be solved to obtain

$$
v(z)=\frac{1-\tanh \left(\left(z+c_{2}\right) / 2 c_{1}\right)^{2}}{c_{1}^{2}} .
$$

Recasting above in its original coordinates, the exact solution of (8) becomes

$$
u(x, y, t)=\frac{1-\tanh \left(\left(y+c_{2}\right) / 2 c_{1}\right)^{2}}{c_{1}^{2} x^{2}}
$$

The graph of this solution is plotted in Figure 3.

Case 5. In this case, we consider the two symmetry generators $\mathbf{X}_{1}, \mathbf{X}_{3}$ which satisfy a commutative relationship $\left[\mathbf{X}_{1}, \mathbf{X}_{3}\right]=0$ as shown in Table 3.

First considering $\mathbf{X}_{1}$, we obtain the similarity variables $r=y, s=x t^{-1 / 2}$, and $w=u$. Equation (8) in these variables reduces to

$$
\begin{aligned}
& s^{2} k(w) w_{s s}+s^{2} k_{w}\left(w_{s}\right)^{2}+s k w_{s}+k_{w}\left(w_{r}\right)^{2} \\
& +k w_{r r}-c N^{2} w+\frac{1}{2} s^{3} w_{s}=0 .
\end{aligned}
$$

First writing $X_{3}$ into $\widehat{X}_{3}=\partial / \partial r$ and solving the resulting characteristic equation the similarity variables are given by $s=z$ and $v(z)=w$. These variables can be used to recast (84) to an ODE,

$$
z^{2} k(v) v_{z z}+z^{2} k_{v}\left(v_{z}\right)^{2}+z k(v) v_{z}-c N^{2} v+\frac{1}{2} z^{3} v_{z}=0
$$

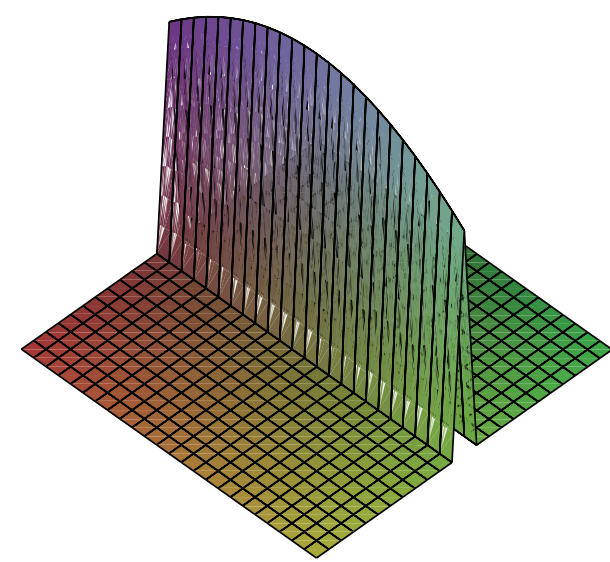

Figure 3

Choosing $k(v)=1$, the solution of (85) becomes

$$
\begin{aligned}
& v(z) \\
& =C_{1} \exp \left(-\frac{1}{8} z^{2}\right) \\
& \times z\left(\operatorname{Bessel} I\left(0, \frac{1}{8} z^{2}\right)+\operatorname{Bessel} I\left(1, \frac{1}{8} z^{2}\right)\right) \\
& +C_{2} \exp \left(-\frac{1}{8} z^{2}\right) \\
& \times z\left(-\operatorname{Bessel} K\left(0, \frac{1}{8} z^{2}\right)+\operatorname{Bessel} K\left(1, \frac{1}{8} z^{2}\right)\right) .
\end{aligned}
$$

Therefore, solution of (8) becomes

$$
\begin{aligned}
u(x, y, t) & C_{1} \exp \left(-\frac{1}{8} \frac{x^{2}}{t}\right) \frac{x}{\sqrt{t}} \\
& \times\left(\operatorname{Bessel} I\left(0, \frac{1}{8} \frac{x^{2}}{t}\right)+\operatorname{Bessel} I\left(1, \frac{1}{8} \frac{x^{2}}{t}\right)\right) \\
& +C_{2} \exp \left(-\frac{1}{8} \frac{x^{2}}{t}\right) \frac{x}{\sqrt{t}} \\
& \times\left(-\operatorname{Bessel} K\left(0, \frac{1}{8} \frac{x^{2}}{t}\right)+\operatorname{Bessel} K\left(1, \frac{1}{8} \frac{x^{2}}{t}\right)\right) .
\end{aligned}
$$

Reduction in all the remaining cases is given in the form of Tables 6, 7, and 8 .

\section{Summary and Discussion}

A complete classification of the Lie point symmetries of the nonlinear fin equation in cylindrical coordinates according to thermal diffusivity and heat transfer coefficient is obtained. Using an exhaustive procedure, the determining equations 
TABle 6: Reduction: Case 1.1.

\begin{tabular}{llll}
\hline Algebra & Reduction & $z$ & $v$ \\
\hline$\left[X_{1}, X_{2}\right]=0$ & $v_{z}+N^{2} c v=0$ & $t$ & $u$ \\
{$\left[X_{1}, X_{3}\right]=0$} & $v_{z z}-\frac{1}{v} v_{z}^{2}+\frac{N^{2} c \beta}{\gamma} v=0$ & $x \cos y$ & $(\beta-u) \exp \left(-N^{2} c t\right)$ \\
{$\left[X_{1}, X_{4}\right]=0$} & $\frac{\gamma}{\beta-v} v_{z z}+\frac{\gamma}{(\beta-v)^{2}} v_{z}^{2}-N^{2} c v=0$ & $x \cos y$ & $u$ \\
{$\left[X_{2}, X_{3}\right]=0$} & $v_{z z}-\frac{1}{v} v_{z}^{2}+\frac{N^{2} c \beta}{\gamma} v=0$ & $x \sin y$ & $(\beta-u) \exp \left(-N^{2} c t\right)$ \\
{$\left[X_{2}, X_{4}\right]=0$} & $\frac{\gamma}{\beta-v} v_{z z}+\frac{\gamma}{(\beta-v)^{2}} v_{z}^{2}-N^{2} c v=0$ & $x \sin y$ & $u$ \\
{$\left[X_{3}, X_{5}\right]=0$} & $z^{2} v_{z z}-\frac{z^{2}}{v} v_{z}^{2}+z v_{z}+\frac{N^{2} c \beta}{\gamma} z^{2} v=0$ & $x$ & $(\beta-u) \exp \left(N^{2} c t\right)$ \\
{$\left[X_{4}, X_{5}\right]=0$} & $\frac{\gamma}{\beta-v} z^{2} v_{z z}+\frac{\gamma}{(\beta-v)^{2}} z^{2} v_{z}^{2}+\frac{\gamma}{\beta-v} z v_{z}-N^{2} c v z^{2}=0$ & $x$ & $u$ \\
\hline
\end{tabular}

TABLE 7: Reduction: Case 1.2.

\begin{tabular}{|c|c|c|c|}
\hline Algebra & Reduction & $z$ & $v$ \\
\hline$\left[X_{1}, X_{2}\right]=0$ & $v_{z}+N^{2} c v=0$ & $t$ & $u$ \\
\hline$\left[X_{1}, X_{3}\right]=X_{1}$ & $v_{z}-2 \gamma \alpha^{(1+\alpha) / \alpha}(2 \alpha+1) v^{(1 / \alpha)+1}+N^{2} c v=0$ & $t$ & $(x \cos y)^{2 \alpha} u$ \\
\hline$\left[X_{1}, X_{4}\right]=0$ & $v_{z z}+\frac{1}{\alpha} \frac{1}{v} v_{z}^{2}=0$ & $x \cos y$ & $\exp \left(N^{2} c t\right) u$ \\
\hline$\left[X_{1}, X_{5}\right]=0$ & $\gamma(\alpha v)^{1 / \alpha} v_{z z}+\gamma(\alpha v)^{(1 / \alpha)-1} v_{z}^{2}-N^{2} c v=0$ & $x \cos y$ & $u$ \\
\hline$\left[X_{2}, X_{3}\right]=X_{2}$ & $2 \gamma \alpha^{(1 / \alpha)+1}(2 \alpha+1) v^{(1 / \alpha)+1}-N^{2} c v-v_{z}=0$ & $t$ & $(x \sin y)^{-2 \alpha} u$ \\
\hline$\left[X_{2}, X_{4}\right]=0$ & $\gamma \alpha^{1 / \alpha} v^{1 / \alpha} v_{z z}+\gamma \alpha^{1 / \alpha} v^{(1 / \alpha)-1} v_{z}^{2}=0$ & $x \sin y$ & $\exp \left(N^{2} c t\right) u$ \\
\hline$\left[X_{3}, X_{4}\right]=0$ & $v_{z z}+\frac{1}{\alpha} v_{z}^{2}+4 \alpha(\alpha+1) v^{(1 / \alpha)+1}=0$ & $y$ & $e^{N^{2} c t} x^{-2 \alpha} u$ \\
\hline$\left[X_{3}, X_{5}\right]=0$ & $\gamma \alpha^{1 / \alpha} v^{1 / \alpha} v_{z z}+\gamma \alpha^{(1 / \alpha)-1} v^{(1 / \alpha)-1} v_{z}^{2}+4 \gamma \alpha^{(1 / \alpha)+1}(\alpha+1) v^{(1 / \alpha)+1}=0$ & $y$ & $x^{-2 \alpha} u$ \\
\hline$\left[X_{3}, X_{6}\right]=0$ & $v_{z}-4 \gamma \alpha^{(1 / \alpha)+1} v^{(1 / \alpha)+1}+N^{2} c v=0$ & $t$ & $x^{-2 \alpha} u$ \\
\hline$\left[X_{5}, X_{6}\right]=0$ & $\gamma z^{2}(\alpha v)^{1 / \alpha} v_{z z}+\gamma z^{2}(\alpha v)^{(1 / \alpha)-1} v_{z}^{2}-N^{2} c z^{2} v=0$ & $x$ & $u$ \\
\hline
\end{tabular}

TABle 8: Reduction: Case 2.2.

\begin{tabular}{|c|c|c|c|}
\hline Algebra & Reduction & $z$ & $v$ \\
\hline$\left[X_{1}, X_{2}\right]=-2 X_{2}$ & $k(v) v_{z z}+k_{v} v_{z}^{2}-N^{2} c v=0$ & $y$ & $u$ \\
\hline$\left[X_{1}, X_{3}\right]=0$ & $z^{2} k(v) v_{z z}+z^{2} k_{v} v_{z}^{2}+z k(v) v_{z}+\frac{1}{2} z^{3} v_{z}-N^{2} c v=0$ & $x t^{-1 / 2}$ & $u$ \\
\hline$\left[X_{2}, X_{3}\right]=0$ & $z^{2} k(v) v_{z z}+z^{2} k_{v} v_{z}^{2}+z k(v) v_{z}-N^{2} c v=0$ & $x$ & $u$ \\
\hline
\end{tabular}

obtained in the process are completely solved for all possible forms of thermal diffusivity and heat transfer. In all cases reduction of the fin equation is performed. In some cases, the nonlinear fin equation is solved for its exact solutions and solutions plotted. As far as symmetry groups are concerned, it is found that the fin equation admits the maximal Lie symmetry group $G\langle 6\rangle$ while the minimal Lie symmetry group is $G\langle 3\rangle$. The other intermediate groups are $G\langle 5\rangle$ and $G\langle 4\rangle$. It is hoped that the nonlinear fin equation may yield interesting results if the study is extended beyond cylindrical symmetry.

\section{Conflict of Interests}

The authors declare that there is no conflict of interests regarding the publication of this paper.

\section{References}

[1] Q. D. Kern and D. A. Kraus, Extended Surface Heat Transfer, McGraw-Hill, New York, NY, USA, 1972.

[2] R. J. Moitsheki, "Steady heat transfer through a radial fin with rectangular and hyperbolic profiles," Nonlinear Analysis: Real World Applications, vol. 12, no. 2, pp. 867-874, 2011.

[3] M. Pakdemirli and A. Z. Sahin, "Group classification of fin equation with variable thermal properties," International Journal of Engineering Science, vol. 42, no. 17-18, pp. 1875-1889, 2004.

[4] M. Pakdemirli and A. Z. Sahin, "Similarity analysis of a nonlinear fin equation," Applied Mathematics Letters, vol. 19, no. 4, pp. 378-384, 2006.

[5] S. Lie, Theorie der Transoformationsgruppen, B. G. Tubner, Leipzig, Germany, 1893. 
[6] G. W. Bluman and S. C. Anco, Symmetry and Integration Methods for Differential Equations, vol. 154 of Applied Mathematical Sciences, Springer, New York, NY, USA, 2002.

[7] G. W. Bluman, A. F. Cheviakov, and S. C. Anco, Applications of Symmetry Methods to Partial Differential Equations, vol. 168 of Applied Mathematical Sciences, Springer, New York, NY, USA, 2010.

[8] G. W. Bluman and S. Kumei, Symmetries and differential equations, vol. 81 of Applied Mathematical Sciences, Springer, New York, NY, USA, 1989.

[9] B. J. Cantwell, Introduction to Symmetry Analysis, Cambridge Texts in Applied Mathematics, Cambridge University Press, Cambridge, UK, 2002.

[10] A. H. Bokhari, A. H. Kara, and F. D. Zaman, "A note on a symmetry analysis and exact solutions of a nonlinear fin equation," Applied Mathematics Letters, vol. 19, no. 12, pp. 13561360, 2006.

[11] R. J. Moitsheki, T. Hayat, and M. Y. Malik, "Some exact solutions of the fin problem with a power law temperature-dependent thermal conductivity," Nonlinear Analysis: Real World Applications, vol. 11, no. 5, pp. 3287-3294, 2010.

[12] R. J. Moitsheki, "Steady one-dimensional heat flow in a longitudinal triangular and parabolic fin," Communications in Nonlinear Science and Numerical Simulation, vol. 16, no. 10, pp. 3971-3980, 2011.

[13] O. O. Vaneeva, A. G. Johnpillai, R. O. Popovych, and C. Sophocleous, "Group analysis of nonlinear fin equations," Applied Mathematics Letters, vol. 21, no. 3, pp. 248-253, 2008.

[14] R. J. Moitsheki and A. Rowjee, "Steady heat transfer through a two-dimensional rectangular straight fin," Mathematical Problems in Engineering, vol. 2011, Article ID 826819, 13 pages, 2011.

[15] D. C. Look Jr., "Two-dimensional fin with non-constant root temperature," International Journal of Heat and Mass Transfer, vol. 32, no. 5, pp. 977-980, 1989.

[16] S. W. Ma, A. I. Behbahani, and Y. G. Tsuei, "Two-dimensional rectangular fin with variable heat transfer coefficient," International Journal of Heat and Mass Transfer, vol. 34, no. 1, pp. 79-85, 1991.

[17] R. J. Moitsheki and C. Harley, "Steady thermal analysis of two-dimensional cylindrical pin fin with a nonconstant base temperature," Mathematical Problems in Engineering, vol. 2011, Article ID 132457, 17 pages, 2011. 


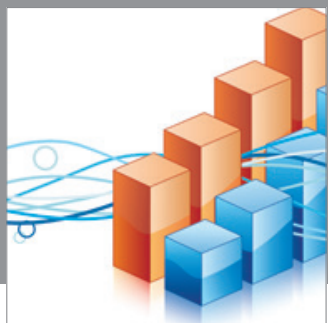

Advances in

Operations Research

mansans

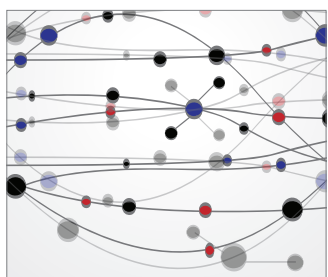

The Scientific World Journal
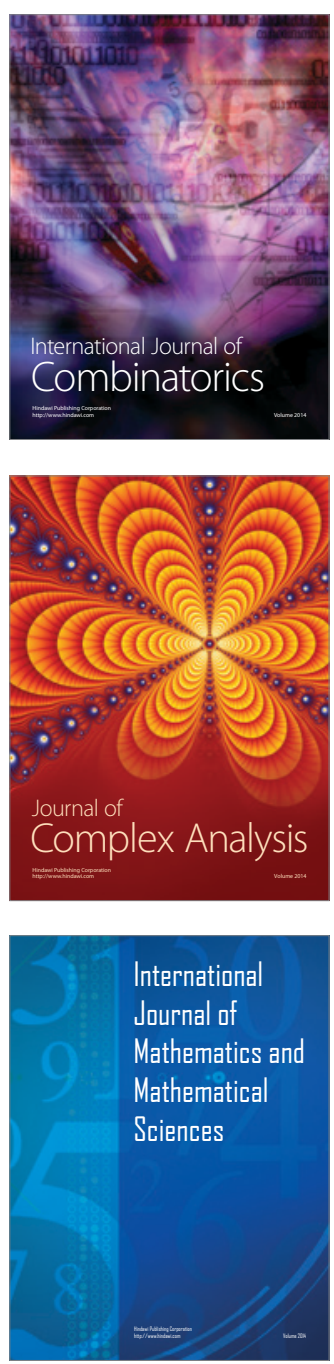
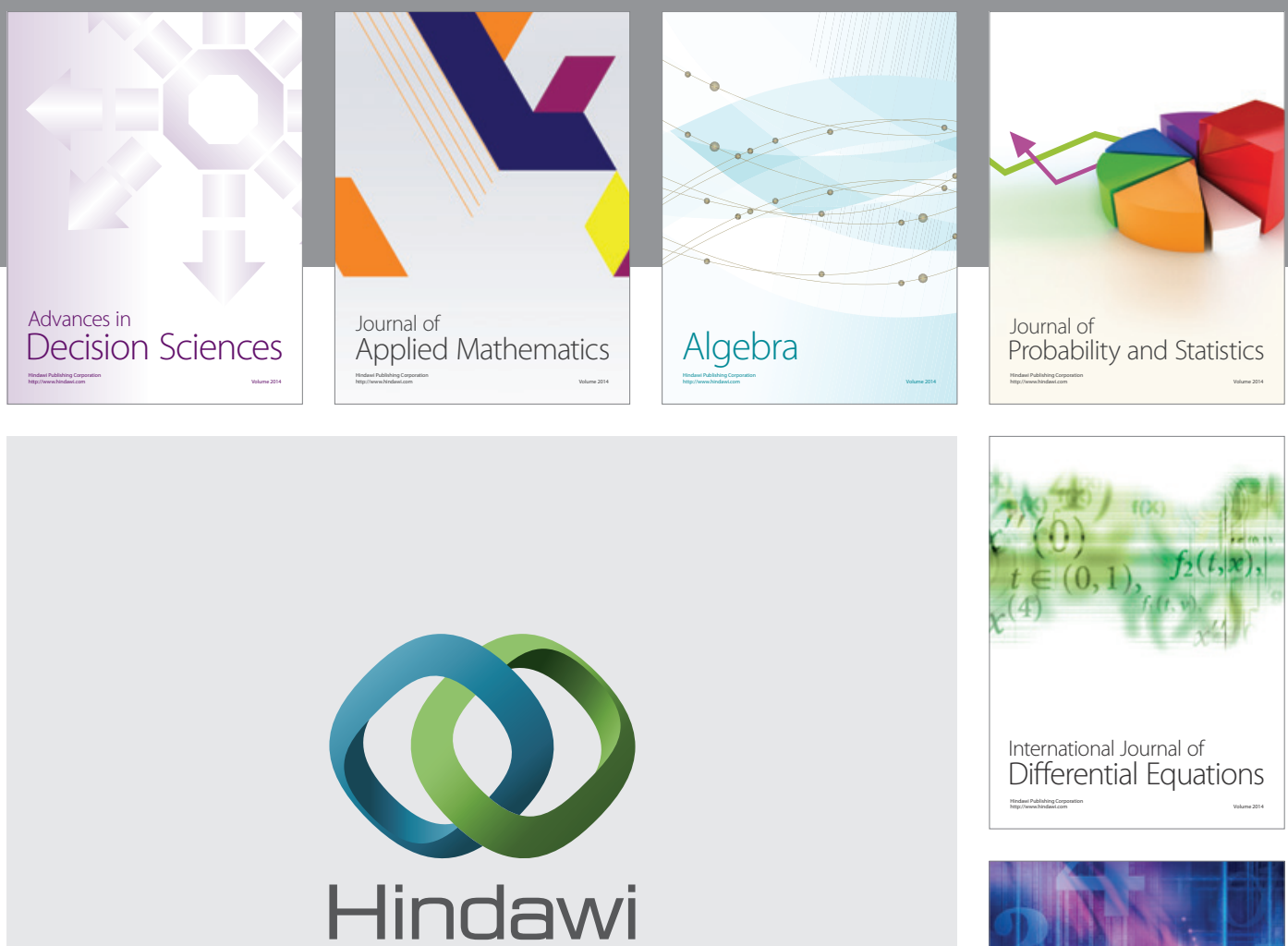

Submit your manuscripts at http://www.hindawi.com
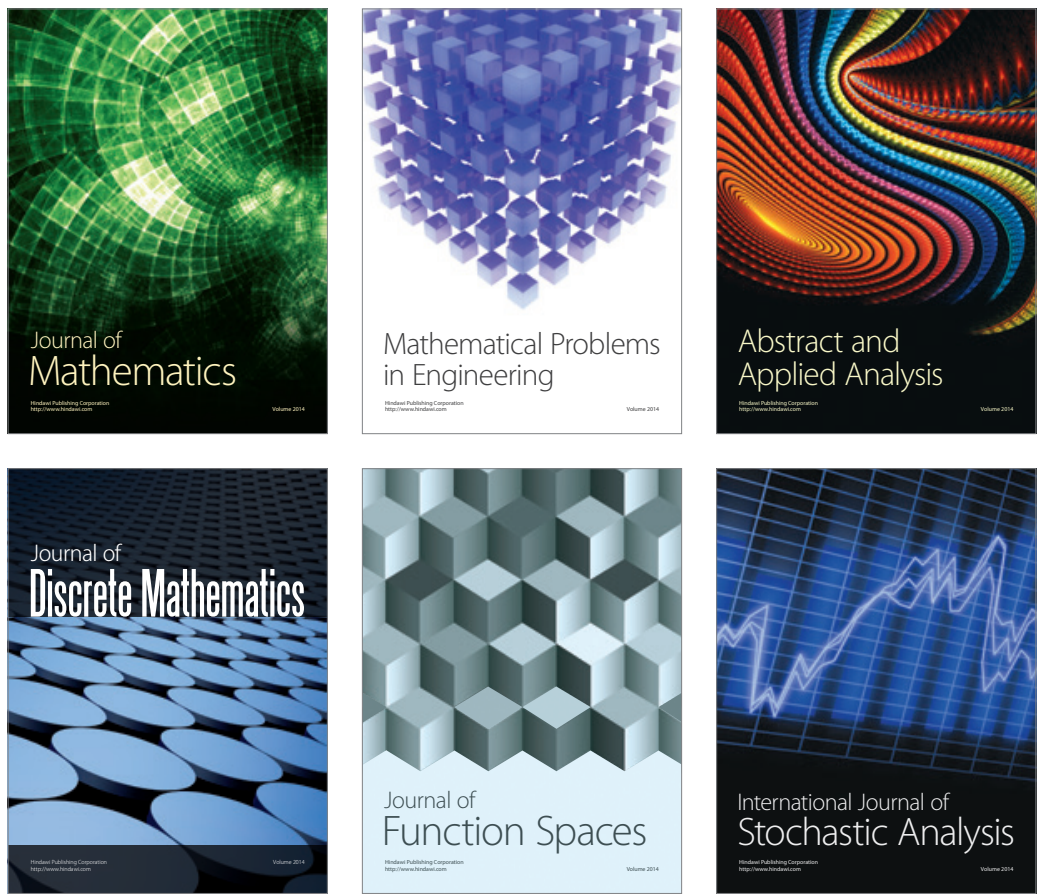

Journal of

Function Spaces

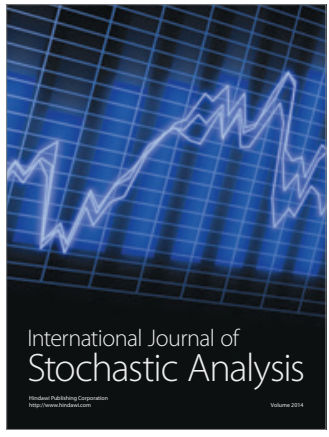

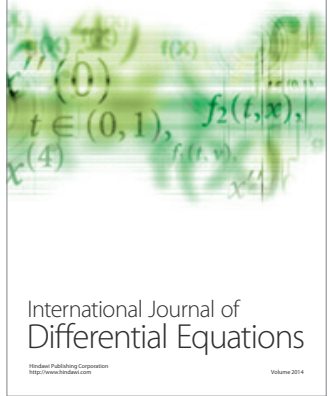
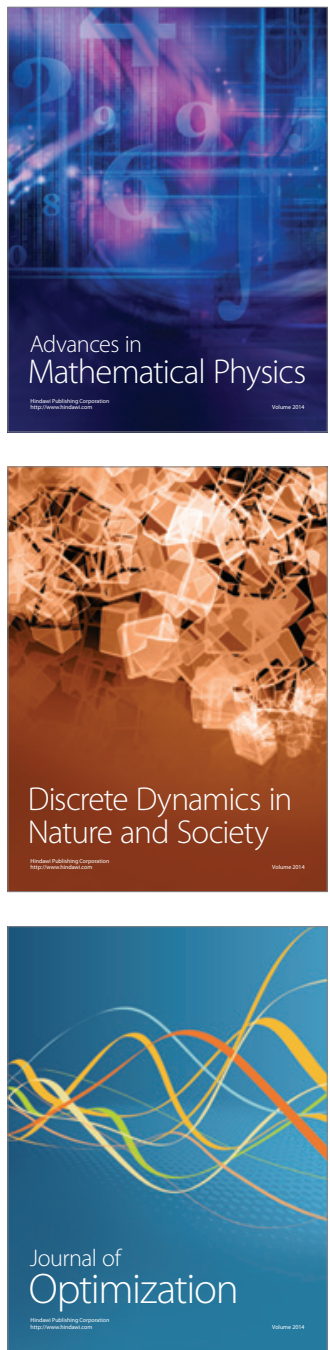